\title{
Optimized Thrust Allocation of Variable-pitch Propellers Quadrotor Control: A Comparative Study on Flip Maneuver
}

\author{
S. R. Nekoo, J. Á. Acosta, A. E. Gomez-Tamm and A. Ollero \\ GRVC Robotics Lab., Departamento de Ingeniería de Sistemas y Automática, Escuela Técnica Superior de Ingeniería, Universidad de \\ Sevilla, Seville, Spain \\ saerafee@yahoo.com, \{jaar, aollero\}@us.es
}

\begin{abstract}
Variable-pitch propellers quadrotors possess nonlinear algebraic relations between force/moment of the system and thrust factors of the rotor dynamics. The nonlinear relations make the thrust allocation a challenging topic in overall control design. The state-dependent Riccati equation (SDRE) is selected as a controller for regulation task in fully coupled six degree-of-freedom (DoF) mode. Common designs of the SDRE fail to deliver a fully coupled six-DoF control due to under-actuation. Virtual constraints are used to deliver a position and orientation control in a cascade design. Within the structure of the SDRE, four thrust allocation methods are proposed to compute the thrust factors based on the output results of control system. Practical implementation has been the main reason to generate such allocations. The use of Mean Value Theorem makes it possible to find an implementable formalism for thrust factors since they can be categorized as non-affine systems. Agile and aggressive maneuver is one of the application of the variable-pitch propellers quadrotors; so, flip maneuver is studied to highlight the advantages of the thrust allocation methods. Analysis of the four methods and comparisons are carried out to present the advantages and disadvantages of the proposed structures.
\end{abstract}

Keywords-SDRE, thrust allocation, optimization, variablepitch, null-space, optimal control, flip maneuver.

\section{INTRODUCTION}

The control of quadrotors has become an interesting topic due to large contribution of the autonomous aerial vehicles (AUVs) in the surveillance, load-carrying, inspection and etc. The state-dependent Riccati equation was used for the first time for quadrotor control by Voos [1]. The under-actuation of the system made the orientation control possible and the quadrotor was regulated to desired attitude with constant motion speed. This problem showed itself in different literature, and forced the design to employ other methods to succeed, such as neural network [2], $\theta$-D based approach [3], and sliding mode design [4].

This current research proposes a new regulation control scheme, based on the SDRE, for a fully coupled six-DoF quadrotor. The attitude of a quadrotor is controllable with a state-dependent coefficient (SDC) parameterization; however, the position control lacks two actuators for $x$ and $y$ direction. The total thrust of the AUV only controls $z$ and distributed into the translational dynamics thorough a rotation matrix. Three virtual constraints are employed to relate the $x y$ planar motion to $z$ or in other words, total thrust. So, the control problem is done based on a fully actuated system (virtual), and then three virtual inputs are transformed to one actual thrust. This approach is so-called cascade design [5], presented for quadrotors. Wang and Jia studied the trajectory tracking problem of a quadrotor and its global stability analysis and control design using cascade theory [5]. Cao and Lynch presented inner-outer loop control for quadrotor UAVs with input and state constraints and used cascade design to determine the desired orientation values to regulate the AUV in fully controlled state [6].

Fixed-pitch propellers quadrotors are much more popular than the variable-pitch ones. The reason is simplicity, more stability, less complexity in mechanism and linear relation between force/moment inputs and rotors' angular velocities. As a result, thrust allocation problem was mostly investigated in marine vessels and autonomous underwater vehicles. Lindegaard and Fossen studied fuel-efficient rudder and propeller control allocation to control a marine craft [7]. The optimization was non-convex due to existence of local minima. Garus presented the thrust allocation problem as an unconstrained optimization for an AUV [8]. The main achievement of the work was the less complexity in computations and more flexibility in structure of a propulsion system. The thrust allocation was also investigated for quadrotors, specifically the variable-pitch ones. Zhou et al. investigated the design of feedback linearization control and reconfigurable control allocation for a quadrotor UAV [9]. The allocation used a linearized map to relate the thrusts to force/moments inputs of Qball-X4 nonlinear model. Sadeghzadeh et al. presented control allocation and reallocation for a modified quadrotor helicopter against actuator faults [10]. The allocation was based on a special linear map, combined with different constants, related to moments of the quadrotor.

This work presented in this paper, investigates the topic of control allocation of variable-pitch propellers quadrotors. The relation of force/moment inputs and thrusts coefficients of the rotors is nonlinear and algebraic; hence, it is classified as a non-affine problem. 
The main contribution of this work is to propose four novel scenarios based on the Mean Value Theorem to solve the thrust allocation problem, suitable for practical implementations.

\section{Controller Design}

Consider a nonlinear time-invariant system, affine-incontrol as

$$
\dot{\mathbf{x}}=\mathbf{f}(\mathbf{x})+\mathbf{g}(\mathbf{x}, \mathbf{u})
$$

where $\mathbf{x} \in \mathbb{R}^{n}$ is state and $\mathbf{u} \in \mathbb{R}^{m}$ is input vector. $\mathbf{f}(\mathbf{x}): \mathbb{R}^{n} \rightarrow \mathbb{R}^{n}$ and $\mathbf{g}(\mathbf{x}, \mathbf{u}): \mathbb{R}^{n} \times \mathbb{R}^{m} \rightarrow \mathbb{R}^{n}$ are piecewise continuous vector-valued functions which satisfy the Lipschitz condition. System (1) is transformed into SDC parameterization

$$
\dot{\mathbf{x}}=\mathbf{A}(\mathbf{x}) \mathbf{x}+\mathbf{B}(\mathbf{x}) \mathbf{u}
$$

where $\mathbf{A}(\mathbf{x}): \mathbb{R}^{n} \rightarrow \mathbb{R}^{n \times n}$ and $\mathbf{B}(\mathbf{x}): \mathbb{R}^{n} \rightarrow \mathbb{R}^{n \times m}$ are designed to generate a completely controllable pair of $\{\mathbf{A}(\mathbf{x}), \mathbf{B}(\mathbf{x})\}$

[11]. The cost function is $J=\frac{1}{2} \int_{0}^{\infty}\left[\mathbf{x}^{T} \mathbf{Q}(\mathbf{x}) \mathbf{x}+\mathbf{u}^{T} \mathbf{R}(\mathbf{x}) \mathbf{u}\right] \mathrm{d} t$ in which $\mathbf{Q}(\mathbf{x}): \mathbb{R}^{n} \rightarrow \mathbb{R}^{n \times n}$ is a weighting matrix for states and $\mathbf{R}(\mathbf{x}): \mathbb{R}^{n} \rightarrow \mathbb{R}^{m \times m}$ is one for inputs. $\mathbf{Q}(\mathbf{x})$ is symmetric positive semi-definite and $\mathbf{R}(\mathbf{x})$ is symmetric positive definite. The pair of $\mathbf{Q}(\mathbf{x})$ and $\mathbf{A}(\mathbf{x})$ in (2), must be a completely observable pair $\left\{\mathbf{A}(\mathbf{x}), \mathbf{Q}^{1 / 2}(\mathbf{x})\right\}$.The common form of SDRE control law is $\mathbf{u}=-\mathbf{R}^{-1}(\mathbf{x}) \mathbf{B}^{T}(\mathbf{x}) \mathbf{K}(\mathbf{x}) \mathbf{x}$, where $\mathbf{K}(\mathbf{x})$ is symmetric positive definite gain, a solution to the SDRE [12]:

$$
\mathbf{A}^{T}(\mathbf{x}) \mathbf{K}(\mathbf{x})+\mathbf{K}(\mathbf{x}) \mathbf{A}(\mathbf{x})-\mathbf{K}(\mathbf{x}) \mathbf{B}(\mathbf{x}) \mathbf{R}(\mathbf{x}) \mathbf{B}^{T}(\mathbf{x}) \mathbf{K}(\mathbf{x})+\mathbf{Q}(\mathbf{x})=\mathbf{0} .
$$

\section{DYNAMICS}

Three translational and three rotational motions must be considered to define six-DoF motion and orientation of a quadrotor. There are two reference frames to establish the generalized coordinates of a quadrotor where Newton's laws of motion are valid. The first reference frame is fixed (earth or inertial) frame: $Z$ from center of earth to surface, $X$ pointing at East and $Y$ pointing at North direction; called East-NorthUp (ENU). The next coordinate (moving coordinate or body frame) is attached to the center-of-mass (CoM) of a quadrotor; the position of the CoM is presented by vector $\left[x_{\mathrm{c}}(t), y_{\mathrm{c}}(t), z_{\mathrm{c}}(t)\right]^{T}(\mathrm{~m})$, illustrated in Fig. 1.

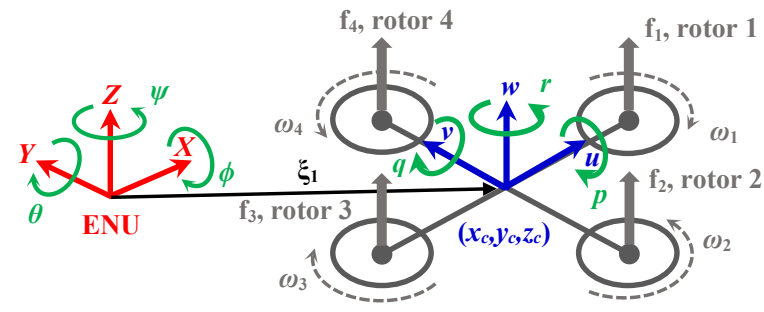

Fig. 1. Fixed and moving reference frame.
The absolute linear position vector of a quadrotor in the inertial frame is $\xi_{1}(t)=\left[x_{\mathrm{c}}(t), y_{\mathrm{c}}(t), z_{\mathrm{c}}(t)\right]^{T}(m)$, the three Euler angles in inertial frame, roll-pitch-yaw, are set in a vector $\xi_{2}(t)=[\phi(t), \theta(t), \psi(t)]^{T}(\mathrm{rad})$, linear velocity vector in body frame is $\mathbf{v}_{1}(t)=[u(t), v(t), w(t)]^{T}(\mathrm{~m} / \mathrm{s})$, and angular velocity vector in body frame is $\mathbf{v}_{2}(t)=[p(t), q(t), r(t)]^{T}(\mathrm{rad} / \mathrm{s})$. The following kinematics relations are held between inertial and body frame [13]:

$$
\begin{gathered}
\dot{\xi}_{1}=\mathbf{R}_{Z Y X}\left(\xi_{2}\right) \mathbf{v}_{1} \\
\dot{\xi}_{2}=\mathbf{T}\left(\xi_{2}\right) \boldsymbol{v}_{2}
\end{gathered}
$$

where $\mathbf{R}_{Z Y X}\left(\xi_{2}\right)$ is found based on multiplication of the three rotation matrices around three main axes:

$$
\begin{gathered}
\mathbf{R}_{Z Y X}\left(\xi_{2}\right)=\left[\begin{array}{ccc}
c_{\theta} c_{\psi} & s_{\phi} s_{\theta} c_{\psi}-c_{\phi} s_{\psi} & c_{\phi} s_{\theta} c_{\psi}+s_{\phi} s_{\psi} \\
c_{\theta} s_{\psi} & s_{\phi} s_{\theta} s_{\psi}+c_{\phi} c_{\psi} & c_{\phi} s_{\theta} s_{\psi}-s_{\phi} c_{\psi} \\
-s_{\theta} & s_{\phi} c_{\theta} & c_{\phi} c_{\theta}
\end{array}\right], \\
\mathbf{T}\left(\xi_{2}\right)=\left[\begin{array}{ccc}
1 & s_{\phi} t_{\theta} & c_{\phi} t_{\theta} \\
0 & c_{\phi} & -s_{\phi} \\
0 & s_{\phi} / c_{\theta} & c_{\phi} / c_{\theta}
\end{array}\right],
\end{gathered}
$$

in which e.g. $c_{\psi}=\cos (\psi(t))$ and $t_{\theta}=\tan (\theta(t))$. The matrix $\mathbf{T}\left(\boldsymbol{\xi}_{2}\right)=\mathbf{W}^{-1}\left(\xi_{2}\right)$ holds in which:

$$
\begin{aligned}
\mathbf{v}_{2} & =\left(\mathbf{I}_{3 \times 3}\left[\begin{array}{c}
\dot{\phi} \\
0 \\
0
\end{array}\right] ; \mathbf{R}_{X}(\phi)\left[\begin{array}{c}
0 \\
\dot{\theta} \\
0
\end{array}\right] ; \mathbf{R}_{X}(\phi) \mathbf{R}_{Y}(\theta)\left[\begin{array}{c}
0 \\
0 \\
\dot{\psi}
\end{array}\right]\right) \\
& =\left[\begin{array}{ccc}
1 & 0 & -s_{\theta} \\
0 & c_{\phi} & c_{\theta} s_{\phi} \\
0 & -s_{\phi} & c_{\theta} c_{\phi}
\end{array}\right] \dot{\xi}_{2}=\mathbf{W}\left(\xi_{2}\right) \dot{\xi}_{2},
\end{aligned}
$$

and $\mathbf{W}\left(\boldsymbol{\xi}_{2}\right)$ is invertible if $\operatorname{det}\left(\mathbf{W}\left(\boldsymbol{\xi}_{2}\right)\right)=\cos (\theta) \neq 0$. There is one input force (thrust) $T_{\mathrm{B}}(t)(\mathrm{N})$, acting in direction of $w$ on $\mathrm{CoM}$ of the quadrotor in Fig. 1, and an input torque vector $\boldsymbol{\tau}_{\mathrm{B}}(t)=\left[\begin{array}{lll}\tau_{\phi}(t) & \tau_{\theta}(t) & \tau_{\psi}(t)\end{array}\right]^{T}(\mathrm{~N} . \mathrm{m})$, acting against three Euler angles $\{\phi(t), \theta(t), \psi(t)\} . T_{\mathrm{B}}(t)$ is defined in body frame and $\tau_{\mathrm{B}}(t)$ is set on inertial frame. A transformation is used to transform the input force from body frame to inertial frame:

$$
\mathbf{F}\left(\boldsymbol{\xi}_{2}(t)\right)=\mathbf{R}_{Z Y X}\left(\boldsymbol{\xi}_{2}\right)\left[\begin{array}{c}
0 \\
0 \\
T_{\mathrm{B}}(t)
\end{array}\right]=\left[\begin{array}{c}
c_{\phi} s_{\theta} c_{\psi}+s_{\phi} s_{\psi} \\
c_{\phi} s_{\theta} s_{\psi}-s_{\phi} c_{\psi} \\
c_{\phi} c_{\theta}
\end{array}\right] T_{\mathrm{B}} .
$$

Lagrange method is chosen to generate equation of motion, so, generalized coordinates are selected as $\mathbf{q}(t)=\left\{x_{\mathrm{c}}(t), y_{\mathrm{c}}(t), z_{\mathrm{c}}(t), \phi(t), \theta(t), \psi(t)\right\}$. Lagrangian function is shaped [14]:

$$
L(\mathbf{q}, \dot{\mathbf{q}})=\frac{1}{2} m \dot{\xi}_{1}^{T} \dot{\xi}_{1}+\frac{1}{2} \mathbf{v}_{2}^{T} \mathbf{I} v_{2}-m g z_{\mathrm{c}},
$$


where $g\left(\mathrm{~m} / \mathrm{s}^{2}\right)$ is gravity acceleration, $m(\mathrm{~kg})$ is total mass of the quadrotor and $\mathbf{I}=\operatorname{diag}\left(I_{x x}, I_{y y}, I_{z z}\right)\left(\mathrm{kgm}^{2}\right)$ is inertia matrix of that in body frame. The quadrotor is supposed to be designed and build symmetrically, as a result, $I_{x x}=I_{y y}$. The term $\mathbf{v}_{2}^{T} \mathbf{I} \boldsymbol{v}_{2}$ in Lagrangian (6), should be transformed to inertial frame. So, using result of Eq. (4), $\boldsymbol{v}_{2}=\mathbf{W}\left(\xi_{2}\right) \dot{\xi}_{2}$, one could find

$$
L(\mathbf{q}, \dot{\mathbf{q}})=\frac{1}{2} m \dot{\xi}_{1}^{T} \dot{\xi}_{1}+\frac{1}{2} \dot{\xi}_{2}^{T} \mathbf{J}\left(\xi_{2}\right) \dot{\xi}_{2}-m g z_{\mathrm{c}}
$$

where $\mathbf{J}\left(\boldsymbol{\xi}_{2}\right)=\mathbf{W}^{T}\left(\boldsymbol{\xi}_{2}\right) \mathbf{I W}\left(\boldsymbol{\xi}_{2}\right)$. Euler-Lagrange equation is [14]:

$$
\frac{\mathrm{d}}{\mathrm{d} t}\left(\frac{\partial L(\mathbf{q}, \dot{\mathbf{q}})}{\partial \dot{\mathbf{q}}}\right)-\frac{\partial L(\mathbf{q}, \dot{\mathbf{q}})}{\partial \mathbf{q}}=\left[\begin{array}{c}
\mathbf{F}\left(\xi_{2}\right) \\
\boldsymbol{\tau}_{\mathrm{B}}
\end{array}\right],
$$

and the linear and angular components are not coupled, so they can be derived independently. Using Lagrangian (7) and deriving the upper set of (8), linear part, result in:

$$
m \ddot{\xi}_{1}+m\left[\begin{array}{l}
0 \\
0 \\
g
\end{array}\right]=\mathbf{F}\left(\xi_{2}\right)=\left[\begin{array}{c}
c_{\phi} s_{\theta} c_{\psi}+s_{\phi} s_{\psi} \\
c_{\phi} s_{\theta} s_{\psi}-s_{\phi} c_{\psi} \\
c_{\phi} c_{\theta}
\end{array}\right] T_{\mathrm{B}},
$$

where generalized force vector is based on (5). The lower set also provides:

$$
\mathbf{J}\left(\xi_{2}\right) \ddot{\xi}_{2}+\frac{\mathrm{d} \mathbf{J}\left(\xi_{2}\right)}{\mathrm{d} t} \dot{\xi}_{2}-\frac{1}{2} \frac{\partial}{\partial \xi_{2}}\left(\dot{\xi}_{2}^{T} \mathbf{J}\left(\xi_{2}\right) \dot{\xi}_{2}\right)=\boldsymbol{\tau}_{\mathrm{B}}
$$

which could be restated as

$$
\mathbf{J}\left(\xi_{2}\right) \ddot{\xi}_{2}+\mathbf{C}\left(\xi_{2}, \dot{\xi}_{2}\right) \dot{\xi}_{2}=\tau_{\mathrm{B}} .
$$
205):

The Coriolis and centrifugal vector is defined ([15], page

$$
\left[\mathbf{C}\left(\boldsymbol{\xi}_{2}, \dot{\boldsymbol{\xi}}_{2}\right) \dot{\boldsymbol{\xi}}_{2}\right]_{3 \times 1}=\sum_{k=1}^{3} \sum_{j=1}^{3}\left(\frac{\partial\left[\mathbf{J}\left(\boldsymbol{\xi}_{2}\right)\right]_{i, j}}{\partial \xi_{k}}-\frac{1}{2} \frac{\partial\left[\mathbf{J}\left(\boldsymbol{\xi}_{2}\right)\right]_{k, j}}{\partial \xi_{i}}\right) \dot{\xi}_{k} \dot{\xi}_{j}, i=1,2,3
$$

where $i$ denotes the $i$-th row of $\left[\mathbf{C}\left(\boldsymbol{\xi}_{2}, \dot{\xi}_{2}\right) \dot{\xi}_{2}\right]$. For each $i$, a $3 \times 3$ cell array is found. A summation turns the $3 \times 3$ cell array into $1 \times 3$ one. Dividing each column of $1 \times 3$ cell to related $\dot{\xi}_{j}$, provides:

$$
\mathbf{C}\left(\xi_{2}, \dot{\xi}_{2}\right)=\left[\begin{array}{ccc}
0 & C_{1,2}\left(\xi_{2}, \dot{\xi}_{2}\right) & C_{1,3}\left(\xi_{2}, \dot{\xi}_{2}\right) \\
C_{2,1}\left(\xi_{2}, \dot{\xi}_{2}\right) & C_{2,2}\left(\xi_{2}, \dot{\xi}_{2}\right) & C_{2,3}\left(\xi_{2}, \dot{\xi}_{2}\right) \\
C_{3,1}\left(\xi_{2}, \dot{\xi}_{2}\right) & C_{3,2}\left(\xi_{2}, \dot{\xi}_{2}\right) & C_{3,3}\left(\xi_{2}, \dot{\xi}_{2}\right)
\end{array}\right],
$$

in which (e.g. $\left.c_{2 \phi}=\cos (2 \phi)\right)$ :

$$
\begin{aligned}
& C_{1,2}\left(\xi_{2}, \dot{\xi}_{2}\right)=\left(I_{y y}-I_{z z}\right)\left(\dot{\theta} s_{2 \phi}-\dot{\psi} c_{2 \phi} c_{\theta}\right) / 2, \\
& C_{1,3}\left(\xi_{2}, \dot{\xi}_{2}\right)=-\dot{\theta}\left(I_{x x} c_{\theta}+c_{\theta}\left(I_{y y}-I_{z z}\right) c_{2 \phi} / 2\right)-\left(I_{y y}-I_{z z}\right) \dot{\psi} c_{\phi} c_{\theta}^{2} s_{\phi}, \\
& C_{2,1}\left(\xi_{2}, \dot{\xi}_{2}\right)=I_{x x} \dot{\psi} c_{\theta} / 2, \\
& C_{2,2}\left(\xi_{2}, \dot{\xi}_{2}\right)=-\left(I_{y y}-I_{z z}\right) s_{2 \phi}\left(4 \dot{\phi}-\dot{\psi} s_{\theta}\right) / 4,
\end{aligned}
$$

$$
\begin{aligned}
C_{2,3}\left(\xi_{2}, \dot{\xi}_{2}\right)= & \dot{\phi}\left(I_{x x} c_{\theta} / 2+\left(I_{y y}-I_{z z}\right) c_{2 \phi} c_{\theta}\right)-\left(I_{y y}-I_{z z}\right) \dot{\theta} s_{2 \phi} s_{\theta} / 4 \\
& +\dot{\psi} c_{\theta} s_{\theta}\left(I_{y y} / 2-I_{x x}+I_{z z} / 2-I_{y y} c_{2 \phi} / 2+I_{z z} c_{2 \phi} / 2\right), \\
C_{3,1}\left(\xi_{2}, \dot{\xi}_{2}\right)= & -I_{x x} \dot{\theta} c_{\theta}, \\
C_{3,2}\left(\xi_{2}, \dot{\xi}_{2}\right)= & \left(I_{y y}-I_{z z}\right)\left(2 \dot{\phi} c_{2 \phi} c_{\theta}-\dot{\theta} s_{2 \phi} s_{\theta}\right) / 2 \\
C_{3,3}\left(\xi_{2}, \dot{\xi}_{2}\right)= & {\left[\dot{\phi} s_{2 \phi}\left(2 I_{y y}-2 I_{z z}\right)\left(c_{2 \theta} / 2+1 / 2\right)\right] / 2 } \\
& -\dot{\theta} s_{2 \theta}\left\{I_{y y} / 2-I_{x x}+I_{z z} / 2-I_{y y} c_{2 \phi} / 2+I_{z z} c_{2 \phi} / 2\right\} .
\end{aligned}
$$

\section{STATE-SPACE REPRESENTATION AND SDC MATRICES}

The position and orientation of the quadrotor are measured in global frame, and the linear and angular velocity of that are measured in local frame. This consideration is based on the assumption $\dot{\xi}_{2}(t) \simeq \mathbf{v}_{2}(t)$ that holds for small angular motions [16], and $\dot{\xi}_{1}(t) \simeq \mathbf{v}_{1}(t)$. The consequence of this assumption is two more approximations $\dot{v}_{1}(t) \simeq \ddot{\xi}_{1}(t)$ and $\dot{v}_{2}(t) \simeq \ddot{\xi}_{2}(t)$. Based on that, the state vector of the system is set

$$
\begin{aligned}
\mathbf{x}(t) & =\left[\xi_{1}^{T}(t), \xi_{2}^{T}(t), \mathbf{v}_{1}^{T}(t), \mathbf{v}_{2}^{T}(t)\right]^{T} \\
& =\left[x_{\mathrm{c}}, y_{\mathrm{c}}, z_{\mathrm{c}}, \phi, \theta, \psi, u, v, w, p, q, r\right]^{T} .
\end{aligned}
$$

Considering state-vector (11), the upper half of the statespace representation of the system uses kinematics relations (3) and (4); and the lower part of that extracts $\ddot{\xi}_{1}$ and $\ddot{\xi}_{2}$ from (9) and (10):

$$
\dot{\mathbf{x}}(t)=\left[\begin{array}{c}
\dot{\xi}_{1}(t) \\
\dot{\boldsymbol{\xi}}_{2}(t) \\
\dot{\mathbf{v}}_{1}(t) \\
\dot{\mathbf{v}}_{2}(t)
\end{array}\right]=\left[\begin{array}{c}
\mathbf{R}_{Z Y X}\left(\boldsymbol{\xi}_{2}\right) \mathbf{v}_{1} \\
\mathbf{T}\left(\boldsymbol{\xi}_{2}\right) \mathbf{v}_{2} \\
1 / m \mathbf{I}_{3 \times 3}\left[\mathbf{R}_{Z Y X, 3}\left(\boldsymbol{\xi}_{2}\right) T_{\mathrm{B}}-m g \mathbf{e}_{3}-\mathbf{D} \dot{\xi}_{1}\right] \\
\mathbf{J}^{-1}\left(\boldsymbol{\xi}_{2}\right)\left[\boldsymbol{\tau}_{\mathrm{B}}-\mathbf{C}\left(\boldsymbol{\xi}_{2}, \dot{\xi}_{2}\right) \dot{\xi}_{2}\right]
\end{array}\right],
$$

where $\mathbf{R}_{Z Y X, 3}\left(\xi_{2}\right)$ is the third column of $\mathbf{R}_{Z Y X}\left(\boldsymbol{\xi}_{2}\right)$ and $\mathbf{e}_{3}=[0,0,1]^{T}$. The aerodynamics effect is incorporated into the dynamics of the system thorough $\mathbf{D}=\operatorname{diag}\left(D_{x}, D_{y}, D_{z}\right)(\mathrm{kg} / \mathrm{s})$ matrix [14]. That is the result of drag force caused by air resistance in which $D_{x}, D_{y}, D_{z}$ are drag coefficients in $(X, Y, Z)$ inertial frame. Based on (12), The SDC parameterization is:

$$
\mathbf{A}_{\mathrm{t}}(\mathbf{x})=\left[\begin{array}{cc}
\mathbf{0}_{3 \times 3} & \mathbf{R}_{Z Y X}\left(\boldsymbol{\xi}_{2}\right) \\
\mathbf{0}_{3 \times 3} & -1 / m \mathbf{I}_{3 \times 3} \mathbf{D}
\end{array}\right], \mathbf{B}_{\mathrm{t}}=\left[\begin{array}{c}
\mathbf{0}_{3 \times 3} \\
1 / m \mathbf{I}_{3 \times 3}
\end{array}\right],
$$

$$
\mathbf{A}_{\mathrm{o}}(\mathbf{x})=\left[\begin{array}{cc}
\mathbf{0}_{3 \times 3} & \mathbf{T}\left(\boldsymbol{\xi}_{2}\right) \\
\mathbf{0}_{3 \times 3} & -\mathbf{J}^{-1}\left(\boldsymbol{\xi}_{2}\right) \mathbf{C}\left(\boldsymbol{\xi}_{2}, \dot{\xi}_{2}\right)
\end{array}\right], \mathbf{B}_{\mathrm{o}}(\mathbf{x})=\left[\begin{array}{c}
\mathbf{0}_{3 \times 3} \\
\mathbf{J}^{-1}\left(\xi_{2}\right)
\end{array}\right],
$$

where index " $\mathrm{t}$ " stands for translation and "o" for orientation.

For the special case of flip maneuver, Eq. (12) is not applicable since the assumption of hovering was regarded for that representation. So, the state vector of the system is changed

$$
\begin{aligned}
\mathbf{x}(t) & =\left[\xi_{1}^{T}(t), \xi_{2}^{T}(t), \dot{\xi}_{1}^{T}(t), \dot{\xi}_{2}^{T}(t)\right]^{T} \\
& =\left[x_{\mathrm{c}}, y_{\mathrm{c}}, z_{\mathrm{c}}, \phi, \theta, \psi, \dot{x}_{\mathrm{c}}, \dot{y}_{\mathrm{c}}, \dot{z}_{\mathrm{c}}, \dot{\phi}, \dot{\theta}, \dot{\psi}\right]^{T},
\end{aligned}
$$

which leads to 


$$
\dot{\mathbf{x}}(t)=\left[\begin{array}{c}
\dot{\xi}_{1}(t) \\
\dot{\boldsymbol{\xi}}_{2}(t) \\
1 / m \mathbf{I}_{3 \times 3}\left[\mathbf{R}_{Z X X, 3}\left(\boldsymbol{\xi}_{2}\right) T_{\mathrm{B}}-m g \mathbf{e}_{3}-\mathbf{D} \dot{\xi}_{1}\right] \\
\mathbf{J}^{-1}\left(\boldsymbol{\xi}_{2}\right)\left[\boldsymbol{\tau}_{\mathrm{B}}-\mathbf{C}\left(\boldsymbol{\xi}_{2}, \dot{\boldsymbol{\xi}}_{2}\right) \dot{\boldsymbol{\xi}}_{2}\right]
\end{array}\right],
$$

with new SDC matrices

$$
\begin{gathered}
\mathbf{A}_{\mathrm{t}}(\mathbf{x})=\left[\begin{array}{cc}
\mathbf{0}_{3 \times 3} & \mathbf{I}_{3 \times 3} \\
\mathbf{0}_{3 \times 3} & -1 / m \mathbf{I}_{3 \times 3} \mathbf{D}
\end{array}\right], \\
\mathbf{A}_{\mathrm{o}}(\mathbf{x})=\left[\begin{array}{cc}
\mathbf{0}_{3 \times 3} & \mathbf{I}_{3 \times 3} \\
\mathbf{0}_{3 \times 3} & -\mathbf{J}^{-1}\left(\boldsymbol{\xi}_{2}\right) \mathbf{C}\left(\boldsymbol{\xi}_{2}, \dot{\xi}_{2}\right)
\end{array}\right] .
\end{gathered}
$$

\section{Virtual CONSTRAint Design}

The error of the state vector is defined as $\mathbf{e}(t)=\mathbf{x}(t)-\mathbf{x}_{\text {des }}$ for regulation. A stable control law is proposed (assuming the system is not under-actuated, Eq. (13)):

$$
\mathbf{U}=-\mathbf{R}_{\mathrm{t}}^{-1}(\mathbf{x}) \mathbf{B}_{\mathrm{t}}^{T}(\mathbf{x}) \mathbf{K}_{\mathrm{t}}(\mathbf{x}) \mathbf{e}_{\mathrm{t}},
$$

where $\mathbf{R}_{\mathrm{t}}(\mathbf{x}): \mathbb{R}^{6} \rightarrow \mathbb{R}^{3 \times 3}$ is the weighting matrix for inputs, $\mathbf{e}_{\mathrm{t}}=\left[\boldsymbol{\xi}_{1}^{T}-\boldsymbol{\xi}_{1, \mathrm{des}}^{T}, \dot{\boldsymbol{\xi}}_{1}^{T}-\dot{\boldsymbol{\xi}}_{\mathrm{l}, \mathrm{des}}^{T}\right]^{T}$ is $\quad$ error $\quad$ vector including translational states and $\mathbf{K}_{\mathrm{t}}(\mathbf{x}): \mathbb{R}^{6} \rightarrow \mathbb{R}^{6 \times 6}$ is the symmetric positive definite solution to the SDRE (dedicated for translational control):

$$
\begin{aligned}
& \mathbf{A}_{\mathrm{t}}^{T}(\mathbf{x}) \mathbf{K}_{\mathrm{t}}(\mathbf{x})+\mathbf{K}_{\mathrm{t}}(\mathbf{x}) \mathbf{A}_{\mathrm{t}}(\mathbf{x}) \\
& \quad-\mathbf{K}_{\mathrm{t}}(\mathbf{x}) \mathbf{B}_{\mathrm{t}}(\mathbf{x}) \mathbf{R}_{\mathrm{t}}^{-1}(\mathbf{x}) \mathbf{B}_{\mathrm{t}}^{T}(\mathbf{x}) \mathbf{K}_{\mathrm{t}}(\mathbf{x})+\mathbf{Q}_{\mathrm{t}}(\mathbf{x})=\mathbf{0} .
\end{aligned}
$$

Two relations could be found as constraints for determining desired values for $\theta$ and $\phi$ [13]:

$$
\begin{aligned}
& \theta_{\mathrm{des}}(t)=\tan ^{-1}\left(\frac{U_{1} \cos \psi_{\mathrm{des}}+U_{2} \sin \psi_{\mathrm{des}}}{U_{3}+g}\right), \\
& \phi_{\mathrm{des}}(t)=\sin ^{-1}\left(\frac{U_{1} \sin \psi_{\mathrm{des}}-U_{2} \cos \psi_{\mathrm{des}}}{\sqrt{U_{1}^{2}+U_{2}^{2}+\left(U_{3}+g\right)^{2}}}\right) .
\end{aligned}
$$

So, the desired vector $\xi_{2, \text { des }}(t)$, is defined as

$$
\xi_{2, \mathrm{des}}(t)=\left[\begin{array}{lll}
\phi_{\mathrm{des}}(t) & \theta_{\mathrm{des}}(t) & \psi_{\mathrm{des}}(t)
\end{array}\right]^{T},
$$

where desired $\psi_{\text {des }}(t)$, in (16)-(18), could be independently set. Consequently, the problem of under-actuation is solved and the thrust is in the form of:

$$
\begin{aligned}
T_{\mathrm{B}}(t) & =m\left\{\left[\mathbf{R}_{Z X X, 3}\left(\xi_{2}\right)\right]_{1} U_{1}+\left[\mathbf{R}_{Z X X, 3}\left(\xi_{2}\right)\right]_{2} U_{2}+\left[\mathbf{R}_{Z X X, 3}\left(\xi_{2}\right)\right]_{3}\left(U_{3}+g\right)\right\} \\
& =m\left[\left(c_{\phi} s_{\theta} c_{\psi}+s_{\phi} s_{\psi}\right) U_{1}+\left(c_{\phi} s_{\theta} s_{\psi}-s_{\phi} c_{\psi}\right) U_{2}+c_{\phi} c_{\theta}\left(U_{3}+g\right)\right] .
\end{aligned}
$$

The design of the rotational control of the quadrotor is straightforward (similar to control law (15)): $\boldsymbol{\tau}_{\mathrm{B}}=-\mathbf{R}_{\mathrm{o}}^{-1}(\mathbf{x}) \mathbf{B}_{\mathrm{o}}^{T}(\mathbf{x}) \mathbf{K}_{\mathrm{o}}(\mathbf{x}) \mathbf{e}_{\mathrm{o}}$, where $\mathbf{R}_{\mathrm{o}}(\mathbf{x}): \mathbb{R}^{6} \rightarrow \mathbb{R}^{3 \times 3}$ is the weighting matrix for inputs, $\mathbf{e}_{\mathrm{o}}=\left[\boldsymbol{\xi}_{2}^{T}-\boldsymbol{\xi}_{2, \mathrm{des}}^{T} \dot{\boldsymbol{\xi}}_{2}^{T}-\dot{\boldsymbol{\xi}}_{2, \mathrm{des}}^{T}\right]^{T}$ is error vector including rotational states and $\mathbf{K}_{\mathrm{o}}(\mathbf{x}): \mathbb{R}^{6} \rightarrow \mathbb{R}^{6 \times 6}$ is the symmetric positive definite solution to the SDRE (dedicated for rotational control, solved by SDC (14)):

$$
\begin{aligned}
& \mathbf{A}_{\mathrm{o}}^{T}(\mathbf{x}) \mathbf{K}_{\mathrm{o}}(\mathbf{x})+\mathbf{K}_{\mathrm{o}}(\mathbf{x}) \mathbf{A}_{\mathrm{o}}(\mathbf{x}) \\
& \quad-\mathbf{K}_{\mathrm{o}}(\mathbf{x}) \mathbf{B}_{\mathrm{o}}(\mathbf{x}) \mathbf{R}_{\mathrm{o}}^{-1}(\mathbf{x}) \mathbf{B}_{\mathrm{o}}^{T}(\mathbf{x}) \mathbf{K}_{\mathrm{o}}(\mathbf{x})+\mathbf{Q}_{\mathrm{o}}(\mathbf{x})=\mathbf{0} .
\end{aligned}
$$

\section{VARIABLE-PITCH ROTOR DYNAMICS}

Equation of blade, a function of thrust coefficient is [17]:

$$
\alpha_{i}(t)=\frac{6 C_{\mathrm{T}_{i}}(t)}{\sigma C_{1_{\alpha}}}+\frac{3}{2} \sqrt{\frac{C_{\mathrm{T}_{i}}(t)}{2}},
$$

where $\alpha_{i}$ and $C_{\mathrm{T}_{i}}$ are blade angle and thrust coefficient of $i$ th rotor with respect, $C_{1_{\alpha}}$ is airfoil lift curve slope, $\sigma=N_{\mathrm{b}} c /(\pi R)$ in which $N_{\mathrm{b}}$ is number of the blades in each rotor, $c(\mathrm{~m})$ is rotor's chord length and $R(\mathrm{~m})$ is the radius of the rotor. Based on the structure of the quadrotor in Fig. 1, the thrust coefficient is related to force/moment inputs of a quadrotor [18] as:

$T_{\mathrm{B}}(t)=\gamma K \sum_{i=1}^{4} C_{\mathrm{T}_{i}}(t)$,

$\tau_{\phi}(t)=\gamma l K\left(C_{\mathrm{T}_{4}}(t)-C_{\mathrm{T}_{2}}(t)\right)$,

$\tau_{\theta}(t)=\gamma l K\left(C_{\mathrm{T}_{3}}(t)-C_{\mathrm{T}_{1}}(t)\right)$,

$\tau_{\psi}(t)=\frac{K R}{\sqrt{2}}\left(-\left|C_{\mathrm{T}_{1}}(t)\right|^{3 / 2}+\left|C_{\mathrm{T}_{2}}(t)\right|^{3 / 2}-\left|C_{\mathrm{T}_{3}}(t)\right|^{3 / 2}+\left|C_{\mathrm{T}_{4}}(t)\right|^{3 / 2}\right)$,

where $\gamma$ is 1 for normal flight and -1 for inverted flight and $K=\rho \pi R^{4} \omega_{\mathrm{ss}}^{2}$ in which $\rho\left(\mathrm{kg} / \mathrm{m}^{3}\right)$ is air density and $\omega_{\mathrm{ss}}(\mathrm{rad} / \mathrm{s})$ is a constant angular velocity of the rotors; $\omega_{\mathrm{ss}}$ is considered constant in variable-pitch flight mode. The minimum angular velocity could be considered as $\omega_{\min }=\sqrt{m g /(4 k)}(\mathrm{rad} / \mathrm{s})$ where $k\left(\mathrm{Ns}^{2} / \mathrm{rad}^{2}\right)$ is lift constant, obtained by an specific angle of blade to keep the quadrotor steady on the air; so, $\omega_{\mathrm{ss}}=\omega_{\min }$.

\section{MAin Result. Control Allocation}

\section{A. Basic Approach}

The relation between the force/moment of the quadrotor and the angular velocities of the rotors are linear in fixed-pitch propellers; however, the same relation with the thrust coefficients in variable-pitch case is nonlinear, Eq. (20). Pretorius and Boje used a mapping approach to define the values of the blade angles, and discussed the limitations such as singularity of thrust in $Z$ direction and fall [19]. Cutler et al. presented a nonlinear differential equation for angular velocity of a variable-pitch propeller, a function of blade angle, and linearized the equation about the hovering condition to analyze the problem [20]. Bhargavapuri et al. presented a control allocation method for solving the nonlinear relation between force/moment inputs and blade angles by computing the derivative of thrust coefficients [17]. The solution to differential equation and solving a nonlinear relation for practical implementation might be a drawback; as a result, in this current work, a solution is used to find the control allocation based on non-affine structure. Reconsider 
(20) when $\mathbf{C}_{\mathrm{T}}(t)=\left[C_{\mathrm{T}_{1}}(t), C_{\mathrm{T}_{2}}(t), C_{\mathrm{T}_{3}}(t), C_{\mathrm{T}_{4}}(t)\right]^{T}$ is factored from that and rewritten as:

$$
\mathbf{C}_{\mathrm{T}}(t)=\mathbf{M}^{-1}\left(\mathbf{C}_{\mathrm{T}}(t)\right)\left[\begin{array}{ll}
T_{\mathrm{B}}(t) & \boldsymbol{\tau}_{\mathrm{B}}^{T}(t)
\end{array}\right]^{T},
$$

where

$$
\mathbf{M}\left(\mathbf{C}_{\mathrm{T}}(t)\right)=\left[\begin{array}{cccc}
\gamma K & \gamma K & \gamma K & \gamma K \\
0 & -\gamma l K & 0 & \gamma l K \\
-\gamma l K & 0 & \gamma l K & 0 \\
-\frac{K R}{\sqrt{2}} \sqrt{\left|C_{\mathrm{T}_{1}}(t)\right|} & \frac{K R}{\sqrt{2}} \sqrt{\left|C_{\mathrm{T}_{2}}(t)\right|} & -\frac{K R}{\sqrt{2}} \sqrt{\left|C_{\mathrm{T}_{3}}(t)\right|} & \frac{K R}{\sqrt{2}} \sqrt{\left|C_{\mathrm{T}_{4}}(t)\right|}
\end{array}\right] .
$$

Solving Eq. (21) is not possible in algebraic form since there exists $\mathbf{C}_{\mathrm{T}}(t)$ in both sides of the equation.

Assumption 1. The vector $\mathbf{f}\left(\mathbf{C}_{\mathrm{T}}(t)\right)=\mathbf{M}\left(\mathbf{C}_{\mathrm{T}}(t)\right) \mathbf{C}_{\mathrm{T}}(t)$ is uniformly continuous smooth vector-valued function in $t \in\left[t_{\mathrm{i}}, t_{\mathrm{f}}\right]$, where $t_{\mathrm{i}}$ and $t_{\mathrm{f}}$ are initial and limited final time. Moreover, $\mathbf{f}\left(t_{\mathrm{i}}\right)$ and $\mathbf{f}\left(t_{\mathrm{f}}\right)$ are uniformly bounded.

Assumption 2. The thrust coefficient possesses positive values $\mathbf{C}_{\mathrm{T}}(t) \in[0, \mathbf{h}]$ in normal flight and is bounded to a constant value $\mathbf{h}$.

Lemma 1. (Mean Value Theorem) [21]. Assume that $\mathbf{f}\left(\mathbf{C}_{\mathrm{T}}(t)\right): \mathbb{R}^{m} \times \mathbb{R}^{\left[t_{\mathrm{i}}, t_{\mathrm{f}}\right]} \rightarrow \mathbb{R}^{m}$ has a derivative at each point of an open set $(\mathbf{a}, \mathbf{b}) \times \mathbb{R}^{\left[\mathrm{t}_{\mathrm{i}}, t_{\mathrm{f}}\right]}$, and assume also $\mathbf{f}$ is continuous at both end points $\mathbf{C}_{\mathrm{T}}\left(t_{\mathrm{i}}\right)=\mathbf{a}$ and $\mathbf{C}_{\mathrm{T}}\left(t_{\mathrm{f}}\right)=\mathbf{b}$. Then there is a vector $\mathbf{C}_{\mathrm{T}}(t) \in(\mathbf{a}, \mathbf{b})$ such that:

$$
\left[\frac{\partial \mathbf{f}\left(\mathbf{C}_{\mathrm{T}}\right)}{\partial \mathbf{C}_{\mathrm{T}}}\right]_{\mathbf{C}_{\mathrm{T}} \in(\mathbf{a}, \mathbf{b})}=\frac{\mathbf{f}(\mathbf{b})-\mathbf{f}(\mathbf{a})}{\mathbf{b}-\mathbf{a}} .
$$

Setting $\mathbf{a}=\mathbf{C}_{\mathrm{T}}\left(t_{\mathrm{i}}\right)$ and $\mathbf{b}=\mathbf{C}_{\mathrm{T}}\left(t_{\mathrm{f}}\right)$, one could present

$$
\mathbf{f}\left(\mathbf{C}_{\mathrm{T}}\left(t_{\mathrm{f}}\right)\right)=\mathbf{f}\left(\mathbf{C}_{\mathrm{T}}\left(t_{\mathrm{i}}\right)\right)+\left[\frac{\partial \mathbf{f}\left(\mathbf{C}_{\mathrm{T}}\right)}{\partial \mathbf{C}_{\mathrm{T}}}\right]_{\mathbf{C}_{\mathrm{T}} \in(\mathbf{a}, \mathbf{b})}\left[\mathbf{C}_{\mathrm{T}}\left(t_{\mathrm{f}}\right)-\mathbf{C}_{\mathrm{T}}\left(t_{\mathrm{i}}\right)\right]
$$

Remark 1. Finding the derivative term between the minimum and maximum bound of interval $\mathbf{C}_{\mathrm{T}}(t) \in(\mathbf{a}, \mathbf{b})$, and $\mathbf{f}\left(\mathbf{C}_{\mathrm{T}}\left(t_{\mathrm{f}}\right)\right)$, Eq. (22), is a difficult task, especially for practical implementation and impose heavy machinery; so the problem is approximated by

$$
\mathbf{f}\left(\mathbf{C}_{\mathrm{T}}(i)\right)=\left[\frac{\partial \mathbf{f}\left(\mathbf{C}_{\mathrm{T}}\right)}{\partial \mathbf{C}_{\mathrm{T}}}\right]_{\mathbf{C}_{\mathrm{T}}(i-1)} \mathbf{C}_{\mathrm{T}}(i) .
$$

Setting the initial time zero $t_{\mathrm{i}}=0$, and considering that each row of $\mathbf{f}\left(\mathbf{C}_{\mathrm{T}}\right)$ has at least one term of $C_{\mathrm{T}_{i}}$, so $\mathbf{f}(\mathbf{0})=\mathbf{0}$. Applying Lemma 1 on Eq. (21) and name $\overline{\mathbf{M}}\left(\mathbf{C}_{\mathrm{T}}\right)$ as

$$
\overline{\mathbf{M}}\left(\mathbf{C}_{\mathrm{T}}\right)=\frac{\partial \mathbf{f}\left(\mathbf{C}_{\mathrm{T}}\right)}{\partial \mathbf{C}_{\mathrm{T}}},
$$

result in

$$
\overline{\mathbf{M}}\left(\mathbf{C}_{\mathrm{T}}\right)=\left[\begin{array}{cccc}
\gamma K & \gamma K & \gamma K & \gamma K \\
0 & -\gamma l K & 0 & \gamma l K \\
-\gamma l K & 0 & \gamma l K & 0 \\
-c_{1} & c_{2} & -c_{3} & c_{4}
\end{array}\right],
$$

in which

$$
c_{i}=\frac{3 K R}{2 \sqrt{2}} \frac{C_{\mathrm{T}_{i}}}{\left|C_{\mathrm{T}_{i}}\right|} \sqrt{\left|C_{\mathrm{T}_{i}}\right|}=\frac{3 K R}{2 \sqrt{2}} \operatorname{sgn} C_{\mathrm{T}_{i}} \sqrt{\left|C_{\mathrm{T}_{i}}\right|} .
$$

In order to implement the modified non-affine form of Eqs. (23) and (24), for practical implementation, $\mathbf{C}_{\mathrm{T}}$ should be computed based on the update of $\mathbf{C}_{\mathrm{T}}$ in previous loop:

$$
\mathbf{C}_{\mathrm{T}}(i)=\overline{\mathbf{M}}^{-1}\left(\mathbf{C}_{\mathrm{T}}(i-1)\right)\left[\begin{array}{l}
T_{\mathrm{B}}(i) \\
\boldsymbol{\tau}_{\mathrm{B}}^{T}(i)
\end{array}\right],
$$

where $i=1, \ldots, t_{\mathrm{f}}$ with time step of $t_{\mathrm{f}} / N$ in which $N$ is the number of the solution loops.

Note: For inverted flight, $\gamma=-1$, Assumption 2 changes the thrust coefficient to negative values $\mathbf{C}_{\mathrm{T}}(t) \in[-\mathbf{h}, 0)$ and imposes corresponding changes to the procedure such as new form for Eq. (19):

$$
\begin{aligned}
& \text { if } C_{\mathrm{T}_{i}}(t) \geq 0 \quad \alpha_{i}(t)=\gamma\left(\frac{6 C_{\mathrm{T}_{i}}(t)}{\sigma C_{1_{\alpha}}}+\frac{3}{2} \sqrt{\frac{\left|C_{\mathrm{T}_{i}}(t)\right|}{2}}\right), \\
& \text { if } C_{\mathrm{T}_{i}}(t)<0 \quad \alpha_{i}(t)=\gamma\left(\frac{6 C_{\mathrm{T}_{i}}(t)}{\sigma C_{\mathrm{I}_{\alpha}}}-\frac{3}{2} \sqrt{\frac{\left|C_{\mathrm{T}_{i}}(t)\right|}{2}}\right) .
\end{aligned}
$$

\section{B. Pseudo Inverse Approach}

Equation (21) is divided to two sections, linear and nonlinear:

$$
\left[\begin{array}{c}
T_{\mathrm{B}}(t) \\
\tau_{\phi}(t) \\
\tau_{\theta}(t)
\end{array}\right]=\left[\begin{array}{cccc}
\gamma K & \gamma K & \gamma K & \gamma K \\
0 & -\gamma l K & 0 & \gamma l K \\
-\gamma l K & 0 & \gamma l K & 0
\end{array}\right]\left[\mathbf{C}_{\mathrm{T}}(t)\right]_{4 \times 1},
$$

$\tau_{\psi}(t)=$

$$
\frac{K R}{\sqrt{2}}\left(-\left|C_{\mathrm{T}_{1}}(t)\right|^{3 / 2}+\left|C_{\mathrm{T}_{2}}(t)\right|^{3 / 2}-\left|C_{\mathrm{T}_{3}}(t)\right|^{3 / 2}+\left|C_{\mathrm{T}_{4}}(t)\right|^{3 / 2}\right) .
$$

If we consider Eq. (25) as

$$
\mathbf{U}_{3 \times 1}(t)=\mathbf{A}_{3 \times 4}\left[\mathbf{C}_{\mathrm{T}}(t)\right]_{4 \times 1},
$$

an optimization problem could be defined, setting the cost function and constraint as

$$
H_{1}\left(\mathbf{C}_{\mathrm{T}}(t), \lambda(t)\right)=\frac{1}{2}\left[\mathbf{C}_{\mathrm{T}}^{T}(t) \mathbf{C}_{\mathrm{T}}(t)\right]-\lambda^{T}(t)\left[\mathbf{A C}_{\mathrm{T}}(t)-\mathbf{U}(t)\right] .
$$

Taking derivative of cost function respect to $\mathbf{C}_{\mathrm{T}}(t)$ and equating that with zero, result in 


$$
\frac{\partial H_{1}\left(\mathbf{C}_{\mathrm{T}}(t), \boldsymbol{\lambda}(t)\right)}{\partial \mathbf{C}_{\mathrm{T}}(t)}=0 \Rightarrow \mathbf{C}_{\mathrm{T}}(t)=\mathbf{A}^{T} \lambda(t) .
$$

Substituting (29) in (27) results in

$$
\mathbf{U}(t)=\mathbf{A} \mathbf{A}^{T} \lambda(t) \Rightarrow \lambda(t)=\left(\mathbf{A} \mathbf{A}^{T}\right)^{-1} \mathbf{U}(t),
$$

which eventually leads to:

$$
\mathbf{C}_{\mathrm{T}}(t)=\mathbf{A}^{T}\left(\mathbf{A} \mathbf{A}^{T}\right)^{-1} \mathbf{U}(t)=\mathbf{A}^{+} \mathbf{U}(t) .
$$

\section{Augmented Pseudo Inverse Approach}

The optimization problem, represented in Eq. (28), neglects the last row of Eq. (20). To amend this drawback, Eq. (26), must be imposed into the design; which is rewritten as:

$$
\frac{\sqrt{2} \tau_{\psi}(t)}{K R}=f\left(\mathbf{C}_{\mathrm{T}}(t)\right)=\mathbf{a}^{T}\left(\mathbf{C}_{\mathrm{T}}(t)\right) \mathbf{C}_{\mathrm{T}}(t),
$$

where

$$
\mathbf{a}\left(\mathbf{C}_{\mathrm{T}}(t)\right)=\left[-\sqrt{\left|C_{\mathrm{T}_{1}}(t)\right|}, \sqrt{\left|C_{\mathrm{T}_{2}}(t)\right|},-\sqrt{\left|C_{\mathrm{T}_{3}}(t)\right|}, \sqrt{\left|C_{\mathrm{T}_{4}}(t)\right|}\right]^{T} .
$$

Consider the second optimization problem as

$$
\begin{aligned}
& H_{2}\left(\mathbf{C}_{\mathrm{T}}(t), \lambda(t)\right)=\frac{1}{2}\left[\mathbf{C}_{\mathrm{T}}^{T}(t) \mathbf{W} \mathbf{C}_{\mathrm{T}}(t)+\right. \\
& \left.W_{\text {yaw }}\left\{\frac{\sqrt{2} \tau_{\psi}(t)}{K R}-f\left(\mathbf{C}_{\mathrm{T}}(t)\right)\right\}^{2}\right]-\lambda^{T}(t)\left[\mathbf{A C}_{\mathrm{T}}(t)-\mathbf{U}(t)\right],
\end{aligned}
$$

which includes the last row of (20) as a mild constraint in the cost function; $\mathbf{W}$ is a weighting matrix for thrusts and $W_{\text {yaw }}$ is a scalar weighing parameter for yaw. Taking derivative of cost function with respect to $\mathbf{C}_{\mathrm{T}}(t)$ and equating that with zero, results in

$$
\begin{aligned}
& \frac{\partial H_{2}\left(\mathbf{C}_{\mathrm{T}}(t), \lambda(t)\right)}{\partial \mathbf{C}_{\mathrm{T}}(t)}=\mathbf{W C}_{\mathrm{T}}(t) \\
& +W_{\text {yaw }}\left\{f\left(\mathbf{C}_{\mathrm{T}}(t)\right)-\frac{\sqrt{2} \tau_{\psi}(t)}{K R}\right\} \frac{\partial f\left(\mathbf{C}_{\mathrm{T}}(t)\right)}{\partial \mathbf{C}_{\mathrm{T}}(t)}-\mathbf{A}^{T} \boldsymbol{\lambda}(t)=0 .
\end{aligned}
$$

The derivative $\frac{\partial f\left(\mathbf{C}_{\mathrm{T}}(t)\right)}{\partial \mathbf{C}_{\mathrm{T}}(t)}$ is rewritten as

$$
\left[\frac{\partial f\left(\mathbf{C}_{\mathrm{T}}(t)\right)}{\partial \mathbf{C}_{\mathrm{T}}(t)}\right]_{4 \times 1}=\mathbf{F}\left(\mathbf{C}_{\mathrm{T}}(t)\right) \mathbf{C}_{\mathrm{T}}(t)
$$

where

$$
\mathbf{F}\left(\mathbf{C}_{\mathrm{T}}(t)\right)=\frac{3}{2} \operatorname{diag}\left(\frac{-\operatorname{sgn} C_{\mathrm{T}_{1}}}{\sqrt{\left|C_{\mathrm{T}_{1}}\right|}}, \frac{\operatorname{sgn} C_{\mathrm{T}_{2}}}{\sqrt{\left|C_{\mathrm{T}_{2}}\right|}}, \frac{-\operatorname{sgn} C_{\mathrm{T}_{3}}}{\sqrt{\left|C_{\mathrm{T}_{3}}\right|}}, \frac{\operatorname{sgn} C_{\mathrm{T}_{4}}}{\sqrt{\left|C_{\mathrm{T}_{4}}\right|}}\right) .
$$

Substituting (31) in (30) results in:

$$
\begin{gathered}
\left\{\mathbf{W}+W_{\text {yaw }}\left\{f\left(\mathbf{C}_{\mathrm{T}}(t)\right)-\frac{\sqrt{2} \tau_{\psi}(t)}{K R}\right\} \mathbf{F}\left(\mathbf{C}_{\mathrm{T}}(t)\right)\right\} \mathbf{C}_{\mathrm{T}}(t)-\mathbf{A}^{T} \lambda(t)=0, \\
\Rightarrow \mathbf{C}_{\mathrm{T}}(t)=\left\{\mathbf{W}+W_{\text {yaw }}\left\{f\left(\mathbf{C}_{\mathrm{T}}(t)\right)-\frac{\sqrt{2} \tau_{\psi}(t)}{K R}\right\} \mathbf{F}\left(\mathbf{C}_{\mathrm{T}}(t)\right)\right\}^{-1} \mathbf{A}^{T} \boldsymbol{\lambda}(t) .
\end{gathered}
$$

Consequently, the final form of $\mathbf{C}_{\mathrm{T}}(t)$ is reached:

$$
\mathbf{C}_{\mathrm{T}}(t)=\left[\mathbf{\Lambda}\left(\mathbf{C}_{\mathrm{T}}(t)\right)\right]^{-1} \mathbf{A}^{T}\left(\mathbf{A}\left[\mathbf{\Lambda}\left(\mathbf{C}_{\mathrm{T}}(t)\right)\right]^{-1} \mathbf{A}^{T}\right)^{-1} \mathbf{U}(t),
$$

where $\quad \boldsymbol{\Lambda}\left(\mathbf{C}_{\mathrm{T}}(t)\right)=\mathbf{W}+W_{\text {yaw }}\left\{f\left(\mathbf{C}_{\mathrm{T}}(t)\right)-\frac{\sqrt{2} \tau_{\psi}(t)}{K R}\right\} \mathbf{F}\left(\mathbf{C}_{\mathrm{T}}(t)\right)$.

To find a solution to non-affine structure of (32), Mean Value Theorem in Lemma 1 is used:

$$
\mathbf{C}_{\mathrm{T}}^{*}(t)=\left[\mathbf{\Lambda}\left(\mathbf{C}_{\mathrm{T}}(t)\right)\right]^{-1} \mathbf{A}^{T}\left(\mathbf{A}\left[\mathbf{\Lambda}\left(\mathbf{C}_{\mathrm{T}}(t)\right)\right]^{-1} \mathbf{A}^{T}\right)^{-1} \mathbf{U}(t),
$$

which represent a practical form for experimental implementation:

$$
\mathbf{C}_{\mathrm{T}}(i)=\left[\mathbf{\Lambda}\left(\mathbf{C}_{\mathrm{T}}(i-1)\right)\right]^{-1} \mathbf{A}^{T}\left(\mathbf{A}\left[\boldsymbol{\Lambda}\left(\mathbf{C}_{\mathrm{T}}(i-1)\right)\right]^{-1} \mathbf{A}^{T}\right)^{-1}\left[\begin{array}{c}
T_{\mathrm{B}}(i) \\
\tau_{\phi}(i) \\
\tau_{\theta}(i)
\end{array}\right],
$$

in which

$$
\boldsymbol{\Lambda}\left(\mathbf{C}_{\mathrm{T}}(i-1)\right)=\mathbf{W}+W_{\text {yaw }}\left\{f\left(\mathbf{C}_{\mathrm{T}}(i-1)\right)-\frac{\sqrt{2} \tau_{\psi}(i)}{K R}\right\} \mathbf{F}\left(\mathbf{C}_{\mathrm{T}}(i-1)\right),
$$

where $i=1, \ldots, t_{\mathrm{f}}$ with time step of $t_{\mathrm{f}} / N$ in which $N$ is the number of the solution loops.

\section{Null-space of Pseudo Inverse Approach}

Null-space of pseudo inverse is covering the redundancy of the solution to pseudo inverse approach in subsection B. Consider the cost function and the constraint as

$$
\begin{aligned}
& H_{3}\left(\mathbf{C}_{\mathrm{T}}(t), \lambda(t)\right)=\frac{1}{2}\left\{\mathbf{C}_{\mathrm{T}}^{T}(t) \mathbf{C}_{\mathrm{T}}(t)-\boldsymbol{\varphi}^{T}\left(\mathbf{C}_{\mathrm{T}}(t)\right) \boldsymbol{\varphi}\left(\mathbf{C}_{\mathrm{T}}(t)\right)\right\} \\
& -\lambda^{T}(t)\left[\mathbf{A} \mathbf{C}_{\mathrm{T}}(t)-\mathbf{U}(t)\right],
\end{aligned}
$$

where $\left[\varphi\left(\mathbf{C}_{\mathrm{T}}(t)\right)\right]_{4 \times 1}$ is a secondary task for optimization, see Ch. 3.5.1 in [22]. The first condition of optimality results in

$$
\begin{aligned}
& \partial H_{3}\left(\mathbf{C}_{\mathrm{T}}(t), \boldsymbol{\lambda}(t)\right) / \partial \mathbf{C}_{\mathrm{T}}(t)=0 \\
& \quad \Rightarrow \mathbf{C}_{\mathrm{T}}(t)=\mathbf{A}^{T} \boldsymbol{\lambda}(t)+\frac{\partial \boldsymbol{\varphi}\left(\mathbf{C}_{\mathrm{T}}(t)\right)}{\partial \mathbf{C}_{\mathrm{T}}(t)} \boldsymbol{\varphi}\left(\mathbf{C}_{\mathrm{T}}(t)\right) .
\end{aligned}
$$

Substituting (33) in (27) provides

$$
\lambda(t)=\left(\mathbf{A A}^{T}\right)^{-1} \mathbf{U}(t)-\left(\mathbf{A} \mathbf{A}^{T}\right)^{-1} \mathbf{A} \frac{\partial \boldsymbol{\varphi}\left(\mathbf{C}_{\mathrm{T}}(t)\right)}{\partial \mathbf{C}_{\mathrm{T}}(t)} \boldsymbol{\varphi}\left(\mathbf{C}_{\mathrm{T}}(t)\right),
$$

and replacing (34) in (33) results in 


$$
\begin{aligned}
\mathbf{C}_{\mathrm{T}}(t) & =\mathbf{A}^{T}\left(\mathbf{A} \mathbf{A}^{T}\right)^{-1} \mathbf{U}(t) \\
& +\left\{\mathbf{I}_{4 \times 4}-\mathbf{A}^{T}\left(\mathbf{A} \mathbf{A}^{T}\right)^{-1} \mathbf{A}\right\} \frac{\partial \boldsymbol{\varphi}\left(\mathbf{C}_{\mathrm{T}}(t)\right)}{\partial \mathbf{C}_{\mathrm{T}}(t)} \boldsymbol{\varphi}\left(\mathbf{C}_{\mathrm{T}}(t)\right) \\
& =\mathbf{A}^{+} \mathbf{U}(t)+\left\{\mathbf{I}_{4 \times 4}-\mathbf{A}^{+} \mathbf{A}\right\} \frac{\partial \boldsymbol{\varphi}\left(\mathbf{C}_{\mathrm{T}}(t)\right)}{\partial \mathbf{C}_{\mathrm{T}}(t)} \boldsymbol{\varphi}\left(\mathbf{C}_{\mathrm{T}}(t)\right) .
\end{aligned}
$$

To integrate the effect of yaw in the optimization, the secondary task is structured as

$$
\begin{aligned}
& \boldsymbol{\varphi}\left(\mathbf{C}_{\mathrm{T}}(t)\right)= \\
& \frac{K R}{\sqrt{2}}\left[-\left|C_{\mathrm{T}_{1}}(t)\right|^{3 / 2}+\left|C_{\mathrm{T}_{2}}(t)\right|^{3 / 2}-\left|C_{\mathrm{T}_{3}}(t)\right|^{3 / 2}+\left|C_{\mathrm{T}_{4}}(t)\right|^{3 / 2}\right],
\end{aligned}
$$

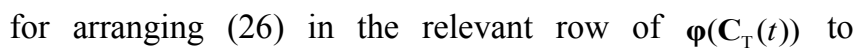
compensate yaw motion. The derivative of (35) is:

$$
\begin{aligned}
& \frac{\partial \boldsymbol{\varphi}\left(\mathbf{C}_{\mathrm{T}}\right)}{\partial \mathbf{C}_{\mathrm{T}}}=\frac{3 K R}{2 \sqrt{2}} \\
& {\left[\begin{array}{cccc}
0 & 0 & 0 & 0 \\
0 & 0 & 0 & 0 \\
0 & 0 & 0 & 0 \\
-\operatorname{sgn} C_{\mathrm{T}_{1}}\left|C_{\mathrm{T}_{1}}\right|^{1 / 2} & \operatorname{sgn} C_{\mathrm{T}_{2}}\left|C_{\mathrm{T}_{2}}\right|^{1 / 2} & -\operatorname{sgn} C_{\mathrm{T}_{3}}\left|C_{\mathrm{T}_{3}}\right|^{1 / 2} & \operatorname{sgn} C_{\mathrm{T}_{4}}\left|C_{\mathrm{T}_{4}}\right|^{1 / 2}
\end{array}\right] .}
\end{aligned}
$$

The practical representation of case for is

$$
\mathbf{C}_{\mathrm{T}}(i)=\mathbf{A}^{+} \mathbf{U}(i)+\left\{\mathbf{I}_{4 \times 4}-\mathbf{A}^{+} \mathbf{A}\right\}\left[\frac{\partial \boldsymbol{\varphi}\left(\mathbf{C}_{\mathrm{T}}(t)\right)}{\partial \mathbf{C}_{\mathrm{T}}(t)}\right]_{t=i-1} \boldsymbol{\varphi}\left(\mathbf{C}_{\mathrm{T}}(i-1)\right) .
$$

\section{SIMULATIONS}

The data of the quadrotor is based on the model in Ref. [14]. The time of simulation was set 10s, the initial condition of the system was set as equilibrium point and the desired position was selected

$$
\mathbf{x}_{\text {des }}\left(t_{\mathrm{f}}\right)=\left[3,-2,1, \phi_{\text {des }}(t), \theta_{\text {des }}(t), 0.3, \mathbf{0}_{1 \times 6}\right]^{T},
$$

in which a flip maneuver starts at $t_{1}=1 \mathrm{~s}$ and should finish the flip before $t_{1}=2 \mathrm{~s}$. After the flip, $t=[2,10] \mathrm{s}$, the quadrotor continues the regulation towards the final condition. The corresponding flip requires the command:

$$
\begin{array}{ccc}
\text { if } \quad t<t_{1} \quad \phi_{\mathrm{des}}(t)=\sin ^{-1}\left(\frac{U_{1} \sin \psi_{\mathrm{des}}-U_{2} \cos \psi_{\mathrm{des}}}{\sqrt{U_{1}^{2}+U_{2}^{2}+\left(U_{3}+g\right)^{2}}}\right), & \phi_{\mathrm{des}}(t)=\pi, \\
\text { if } \quad t_{1}<t<t_{2} & \\
\text { if } & t_{2}<t<t_{\mathrm{f}} & \phi_{\mathrm{des}}(t)=\sin ^{-1}\left(\frac{U_{1} \sin \psi_{\mathrm{des}}-U_{2} \cos \psi_{\mathrm{des}}}{\sqrt{U_{1}^{2}+U_{2}^{2}+\left(U_{3}+g\right)^{2}}}\right) .
\end{array}
$$

The weighting matrices were selected as $\mathbf{W}=\mathbf{I}_{4 \times 4}$, $W_{\mathrm{YAW}}=1 \quad, \quad \mathbf{R}_{\mathrm{o}}=10 \times \mathbf{I}_{3 \times 3} \quad, \quad \mathbf{R}_{\mathrm{t}}=\mathbf{I}_{3 \times 3} \quad, \quad \mathbf{Q}_{\mathrm{t}}=\mathbf{I}_{6 \times 6} \quad$ and $\mathbf{Q}_{\mathrm{o}}=\operatorname{diag}\left(\mathbf{1}_{1 \times 3}, \mathbf{0}_{1 \times 3}\right)$. The states that represent the position of the quadrotor in Cartesian coordinate are shown in Fig. 2. The orientation of the quadrotor is presented in Fig. 3. Linear and angular velocities of the system are illustrated in Figs. 4 and
5. The SDRE trajectory, considering flip, and configuration of the quadrotor are depicted in Fig. 6.

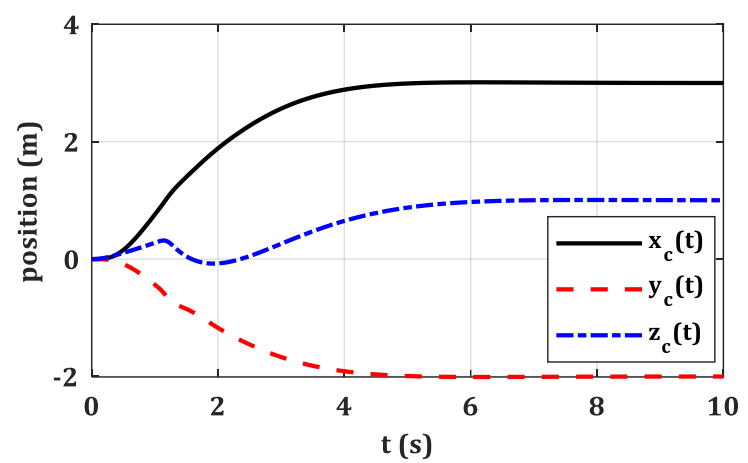

Fig. 2. The states that represent the position of the quadrotor in Cartesian coordinate.

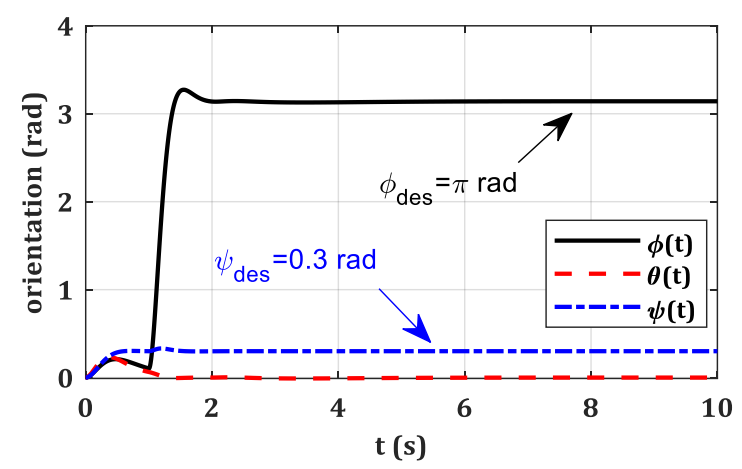

Fig. 3. The orientation of the quadrotor.

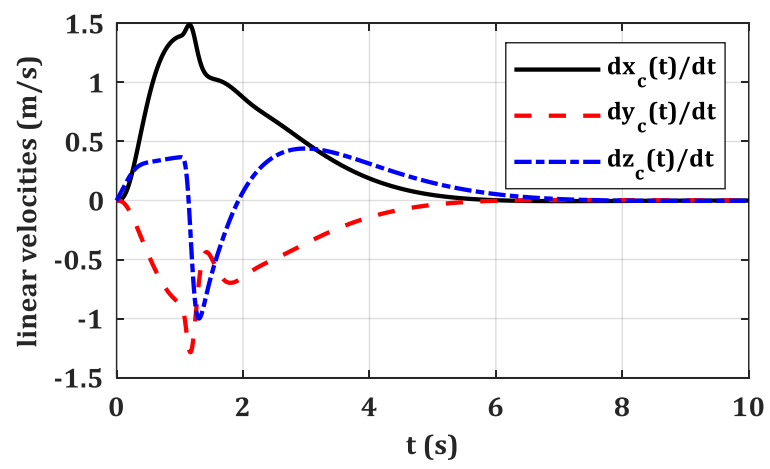

Fig. 4. Linear velocities of the system.

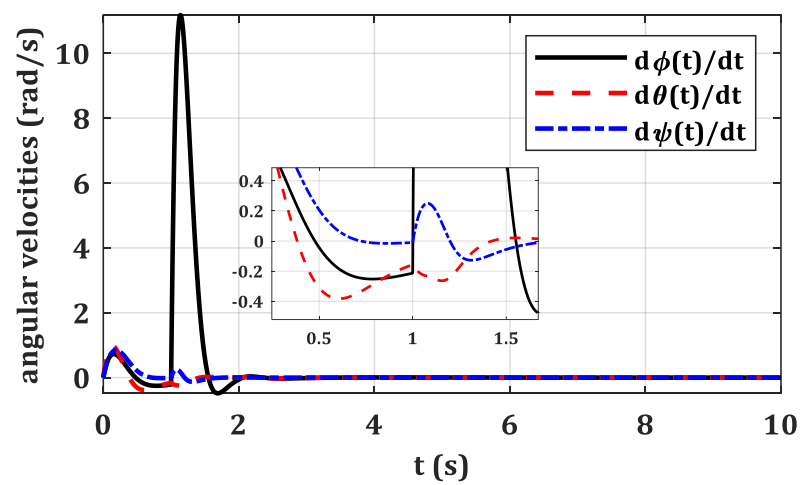

Fig. 5. Angular velocities of the quadrotor. 
(a)

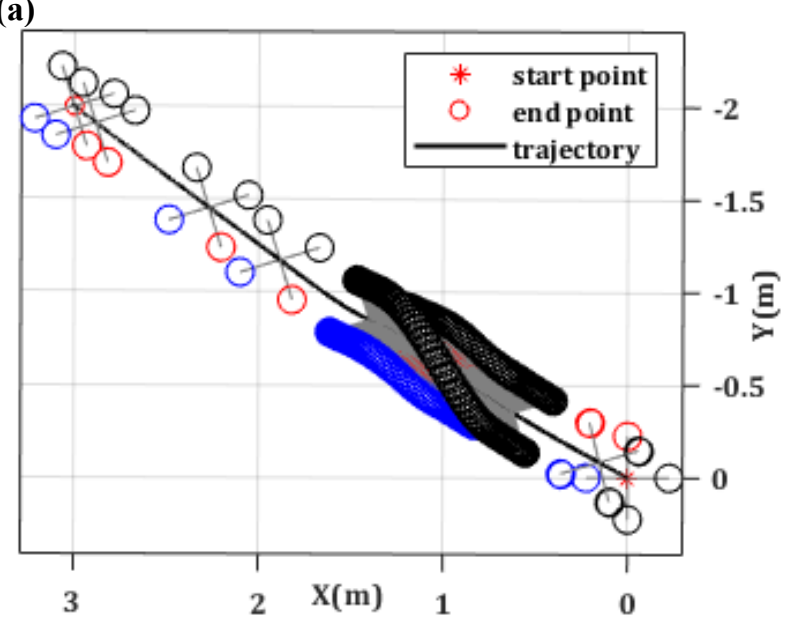

(b)

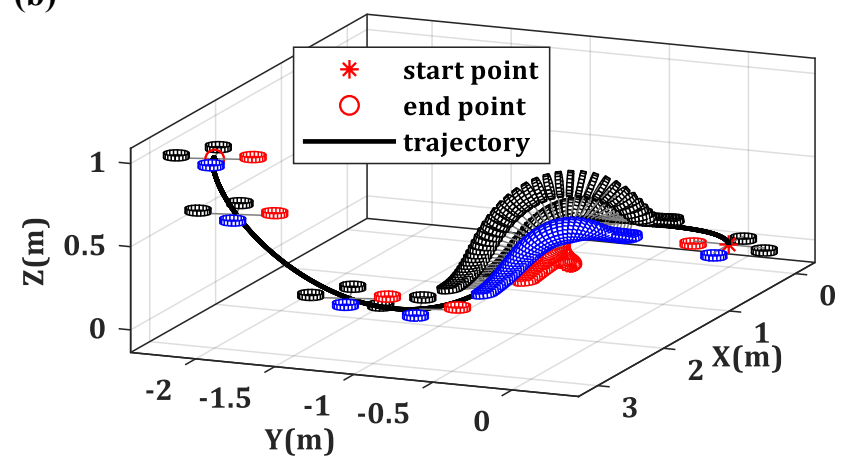

Fig. 6. The SDRE trajectory, considering $\pi$ rad flip, and configuration of the quadrotor; (a) top view, (b) 3D view.

The trust of the system is shown in Fig. 7 and the input moments of the system are presented in Fig. 8. Thrusts of the first and second rotor are shown in Figs. 9 and 10; and the angles of the blades are illustrated in Figs. 11-14. The proposed approach has not limitation in amount of flip as $2 \pi$ rad flip and flip/flip-back were performed, presented in Fig. 15 and 16.

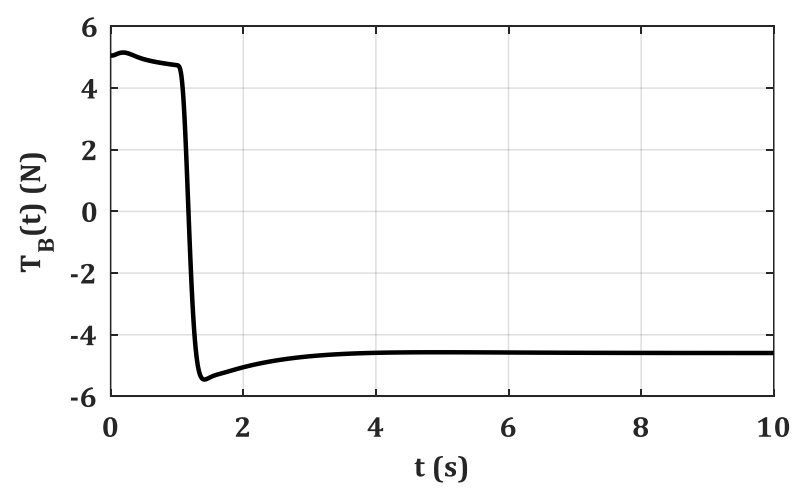

Fig. 7. Thrust of the quadrotor.

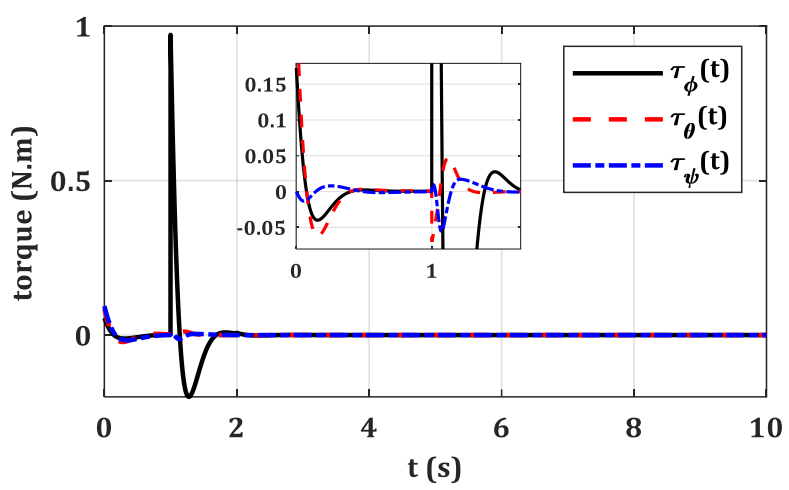

Fig. 8. Input moments of the system.

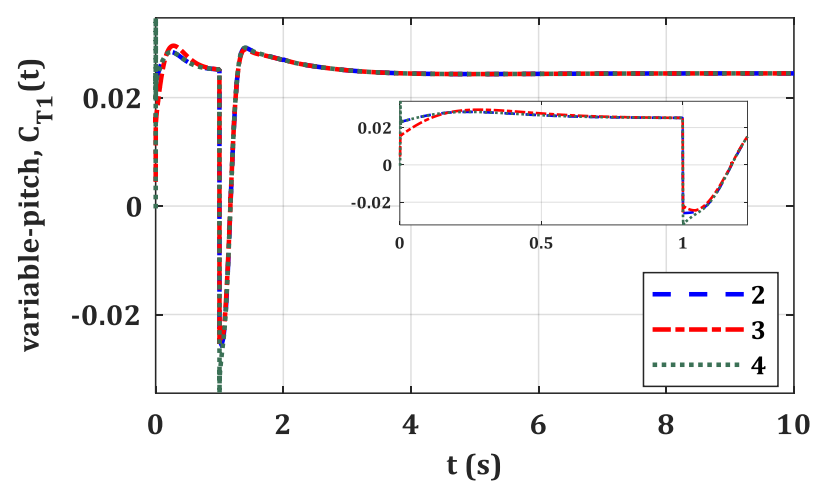

Fig. 9. Thrust factor of the first rotor; (2) pseudo inverse approach (3) augmented pseudo inverse approach (4) null-space of pseudo inverse approach.

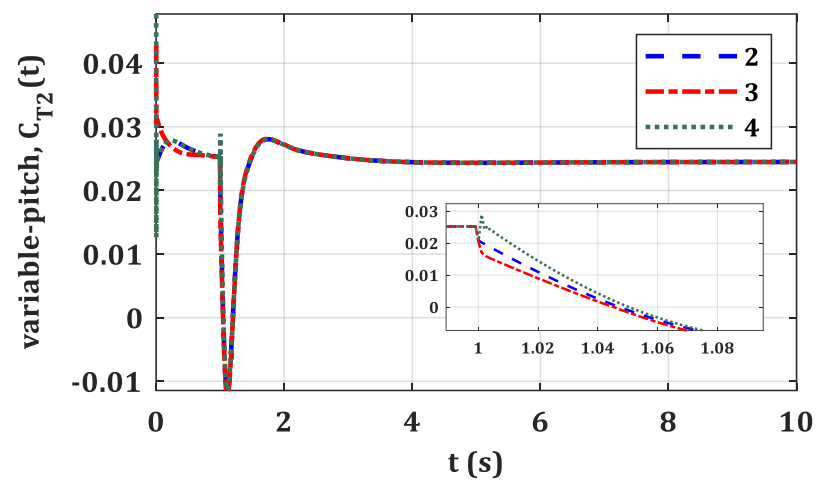

Fig. 10. Thrust factor of the second rotor.

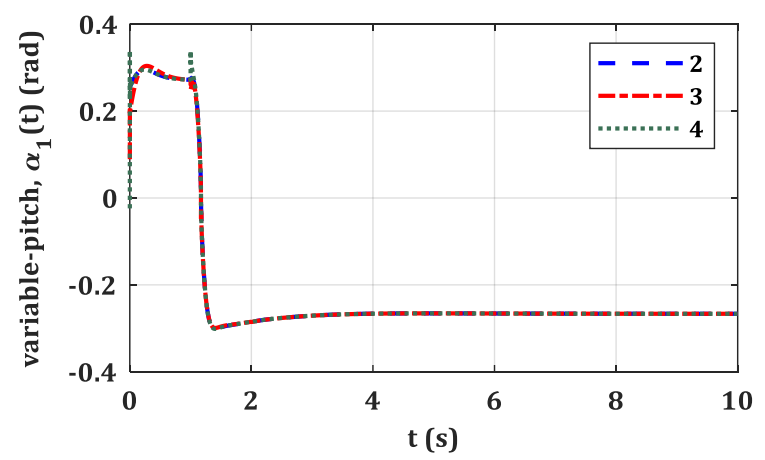

Fig. 11. Angle of the blade, first rotor. 


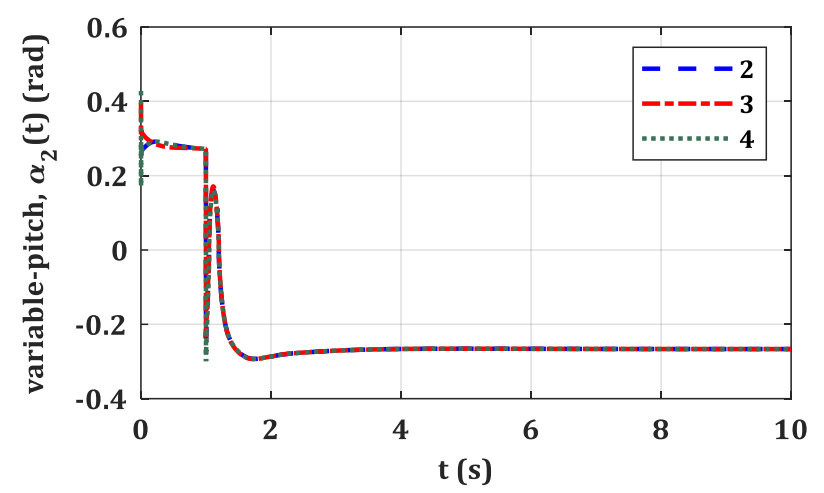

Fig. 12. Angle of the blade, second rotor.

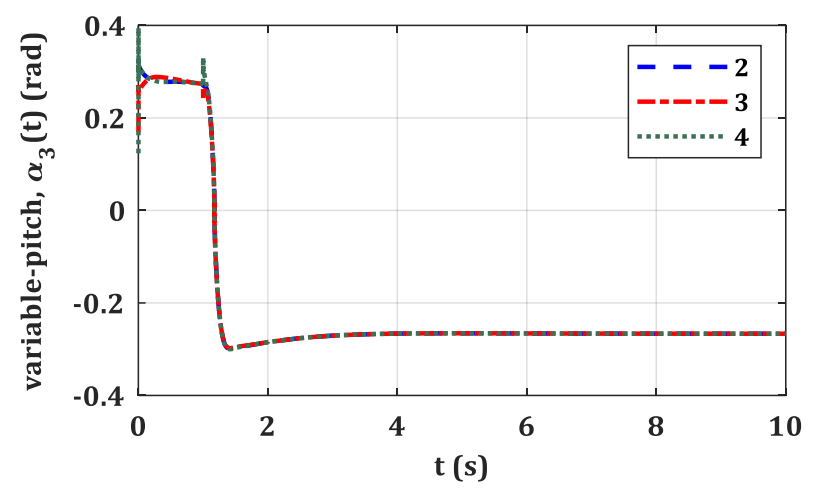

Fig. 13. Angle of the blade, third rotor.

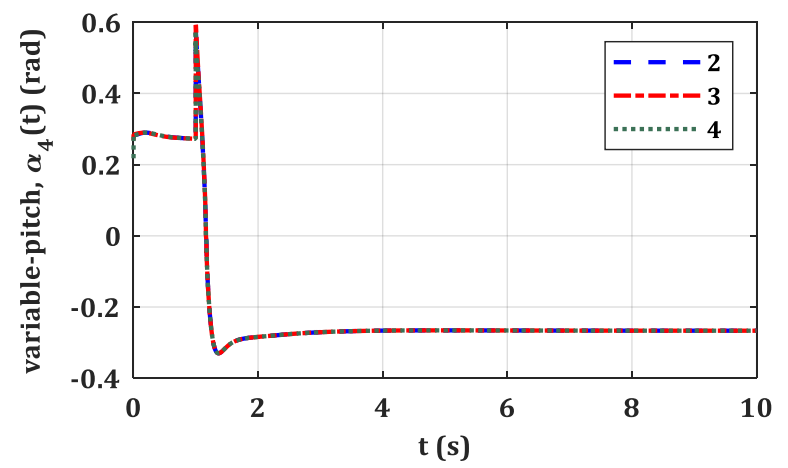

Fig. 14. Angle of the blade, fourth rotor.

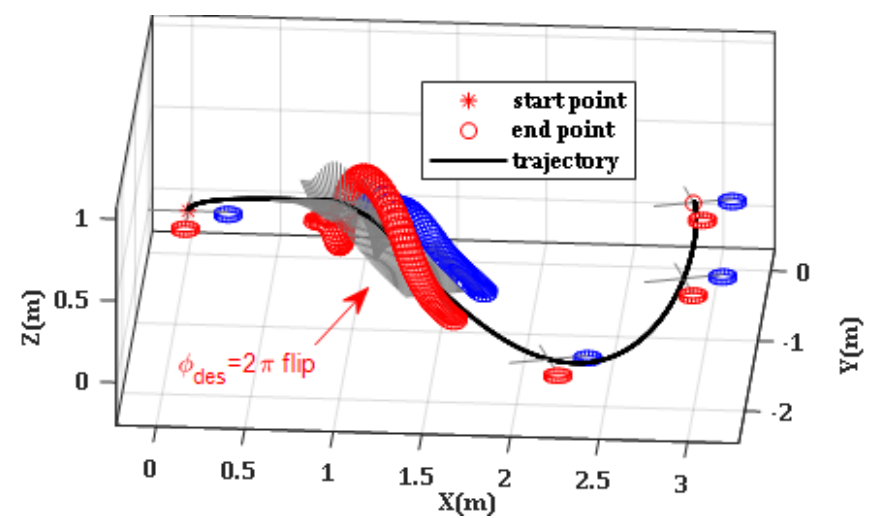

Fig. 15. The SDRE trajectory, considering $2 \pi$ rad flip, and configuration of the quadrotor.

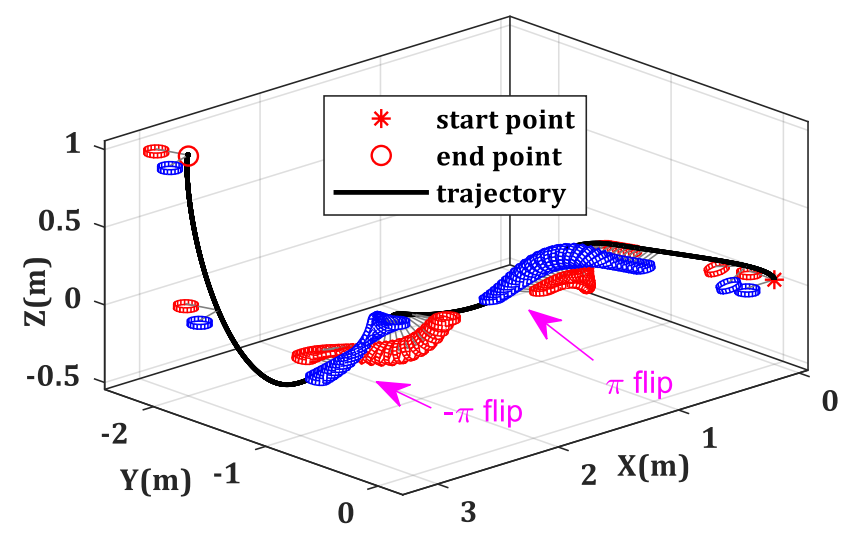

Fig. 16. The SDRE trajectory, considering $\pi$ and $-\pi$ rad flip.

Discussion: The regulation error of the system was found $1.24 \mathrm{~mm}$, Fig. 6. which is precise with respect to travel distance. The error could be even more precise by increasing weighting matrix $\mathbf{Q}_{t}$, or using new form $\mathbf{Q}_{t}=\operatorname{diag}\left(\mathbf{1}_{1 \times 3}, \mathbf{0}_{1 \times 3}\right)$ with the cost of having a little overshoot. A regulation without overshot is also achievable by $\mathbf{Q}_{\mathrm{t}}=\operatorname{diag}\left(\mathbf{a}_{1 \times 3}, \mathbf{b}_{1 \times 3}\right)$ where $\mathbf{b}>\mathbf{a}$. Using Mean Value Theorem without optimization, basic approach, was not depicted in the results due to excessive chattering in Figs. 9-14. The reason is computational difficulty for finding inverse of $\overline{\mathbf{M}}\left(\mathbf{C}_{\mathrm{T}}\right)$ in (24). If one removes $\operatorname{sgn} C_{\mathrm{T}_{i}}$ from $\overline{\mathbf{M}}\left(\mathbf{C}_{\mathrm{T}}\right)$, this approach will results almost similar to other ones. Here in this current research, considering $\operatorname{sgn} C_{\mathrm{T}_{i}}$ in the thrust allocation method, two approaches successfully fulfilled this flip case. The methods are optimal and possess a practical form for experimental implementation: $\mathbf{C}_{\mathrm{T}}(i)=h\left(\mathbf{C}_{\mathrm{T}}(i-1)\right)$. Second method is not considering $\psi$ in the allocation process, so for cases with desired $\psi \neq 0$, the method is indifferent towards it and that is a drawback.

\section{CONCLUSIONS}

This work focused on the thrust allocation problem for variable-pitch propellers quadrotor control based on the statedependent Riccati equation controller. Four methods were suggested for allocation process. The first one used the Mean Value Theorem to solve the allocation preserving the nonlinear algebraic nature of the relation. The second one led to pseudo inverse, the third approach was an optimization to provide a tradeoff between the yaw (nonlinear part) and the linear part in the thrust/input relation and the last one was based on the null-space of the pseudo inverse approach. All four allocation methods have been simulated and compared. Quadrotors are usually performing the regulation and tracking task in hovering state, that means as less variation in attitude as possible. However, multi-rotors with variable-pitch achieve more maneuverability and agile regulation (such as flip maneuver). The relevant state-space representation for flip maneuver (without assumption of hovering) was simulated. Mean Value Theorem provided a practical point of view to overcome the nan-affine structure of nonlinear algebraic allocation equation. 


\section{ACKNOWLEDGMENT}

This work is supported by the HYFLIER project (HYbrid FLying-rolling with-snakE-aRm robot for contact inspection) funded by the European Commission H2020 Programme under grant agreement ID: 779411 (https://cordis.europa.eu/project/rcn/213049).

\section{REFERENCES}

[1] H. Voos, "Nonlinear state-dependent Riccati equation control of a quadrotor UAV," in IEEE Computer Aided Control System Design, IEEE International Conference on Control Applications, IEEE International Symposium on Intelligent Control, Munich, Germany, 2006, pp. 2547-2552.

[2] H. Voos, "Nonlinear and neural network-based control of a small four-rotor aerial robot," in IEEE/ASME international conference on Advanced intelligent mechatronics, ETH Zürich, Switzerland 2007, pp. 1-6.

[3] M. Navabi and H. Mirzaei, " $\theta$-D based nonlinear tracking control of quadcopter," in 4th International Conference on Robotics and Mechatronics, Tehran, 2016, pp. 331-336.

[4] R. Babaie and A. F. Ehyaie, "Robust optimal motion planning approach to cooperative grasping and transporting using multiple UAVs based on SDRE," Transactions of the Institute of Measurement and Control, vol. 39, pp. 1391-1408, 2017.

[5] L. Wang and H. Jia, "The trajectory tracking problem of quadrotor UAV: Global stability analysis and control design based on the cascade theory," Asian Journal of Control, vol. 16, pp. 574-588, 2014

[6] N. Cao and A. F. Lynch, "Inner-outer loop control for quadrotor UAVs with input and state constraints," IEEE Transactions on Control Systems Technology, vol. 24, pp. 1797-1804, 2015.

[7] K.-P. Lindegaard and T. I. Fossen, "Fuel-efficient rudder and propeller control allocation for marine craft: Experiments with a model ship," IEEE Transactions on Control Systems Technology, vol. 11, pp. 850-862, 2003.

[8] J. Garus, "Optimization of thrust allocation in the propulsion system of an underwater vehicle," International Journal of Applied Mathematics and Computer Science, vol. 14, pp. 461467, 2004.

[9] Q.-L. Zhou, Y. Zhang, C.-A. Rabbath, and D. Theilliol, "Design of feedback linearization control and reconfigurable control allocation with application to a quadrotor UAV," in Conference on Control and Fault-Tolerant Systems, Nice, France, 2010, pp. 371-376.
[10] I. Sadeghzadeh, A. Chamseddine, Y. Zhang, and D. Theilliol, "Control allocation and re-allocation for a modified quadrotor helicopter against actuator faults," IFAC Proceedings Volumes, vol. 45, pp. 247-252, 2012.

[11] S. R. Nekoo and M. Irani Rahaghi, "Recursive approximate solution to time-varying matrix differential Riccati equation: linear and nonlinear systems," International Journal of Systems Science, vol. 49, pp. 2797-2807, 2018.

[12] A. Prach, O. Tekinalp, and D. Bernstein, "Nonlinear aircraft flight control using the forward propagating Riccati equation," in AIAA Guidance, Navigation, and Control Conference, San Diego, California, USA, 2016, pp. 13831396.

[13] Z. Zuo, "Trajectory tracking control design with commandfiltered compensation for a quadrotor," IET control theory \& applications, vol. 4, pp. 2343-2355, 2010.

[14] T. Luukkonen, "Modelling and control of quadcopter," Independent research project in applied mathematics, Espoo, vol. 22, 2011.

[15] R. J. Schilling, Fundamentals of robotics: Analysis and control. New Delhi: Prentice-Hall of India Pvt. Ltd., 2003.

[16] A. Das, K. Subbarao, and F. L. Lewis, "Dynamic inversion with zero-dynamics stabilisation for quadrotor control," IET control theory \& applications, vol. 3, pp. 303-314, 2009.

[17] M. Bhargavapuri, S. R. Sahoo, and M. Kothari, "Robust nonlinear control of a variable-pitch quadrotor with the flip maneuver," Control Engineering Practice, vol. 87, pp. 26-42, 2019.

[18] A. K. Shastry, M. T. Bhargavapuri, M. Kothari, and S. R. Sahoo, "Quaternion based adaptive control for package delivery using variable-pitch quadrotors," in Indian Control Conference, 2018, pp. 340-345.

[19] A. Pretorius and E. Boje, "Design and modelling of a quadrotor helicopter with variable pitch rotors for aggressive manoeuvres," IFAC Proceedings Volumes, vol. 47, pp. 12208-12213, 2014.

[20] M. Cutler, N.-K. Ure, B. Michini, and J. How, "Comparison of fixed and variable pitch actuators for agile quadrotors," in AIAA Guidance, Navigation, and Control Conference, 2011, pp. 6406-6423.

[21] M. H. Korayem and S. R. Nekoo, "Finite-time statedependent Riccati equation for time-varying nonaffine systems: Rigid and flexible joint manipulator control," ISA Transactions, vol. 54, pp. 125-144, 2015.

[22] B. Siciliano, L. Sciavicco, L. Villani, and G. Oriolo, Robotics: modelling, planning and control: Springer Science \& Business Media, 2010. 